A 65-year-old man was admitted to hospital with obstructive jaundice. Three years previously he had been diagnosed with pulmonary tuberculosis. During his antituberculous treatment he was found to have a $10-\mathrm{cm}$ cystic mass in the anterior mediastinum that was increasing in size. Computed tomography-guided biopsy showed this to be an epithelial neoplasm with neuroendocrine differentiation. This was surgically removed and pathological examination revealed a moderately differentiated neuroendocrine carcinoma of the thymus. There was focal involvement of the resection margin and lymphovascular spread. He was given postoperative adjuvant radiotherapy and remained well for 2 years.

After admission, ultrasound examination showed a $4-\mathrm{cm}$ mass at the pancreatic head with common bile duct dilatation. Endoscopic retrograde cholangiopancreatography showed a distal common bile duct stricture. Subsequent computed tomography showed a heterogeneously enhanced, 4-cm mass at the head of pancreas, together with multiple necrotic hepatoduodenal nodes (Figure 1). The features were highly suggestive of pancreatic carcinoma. Endoscopic ultrasound-guided fine-needle aspiration was performed and bloody aspirate was obtained (Figure 2).

Cytology examination showed atypical cells with enlarged nuclei with a stippled chromatin pattern, and a small to moderate amount of cytoplasm (Figure $\mathbf{3} \mathbf{a}$ ). Immunostaining demonstrated that these cells were positive for cytokeratins, including AE1/3 and CK7, and the neuroendocrine markers, chromogranin and synaptophysin (Figure $\mathbf{3 b}$ ). The cytologic morphology was identical to that of the previously resected thymic cancer. A diagnosis of metastatic thymic neuroendocrine carcinoma was made, and he was referred for chemotherapy.

Thymic neuroendocrine carcinomas are rare and encompass a wide spectrum of

\title{
Metastatic thymic neuroendocrine carcinoma presenting as a pancreatic tumor
}

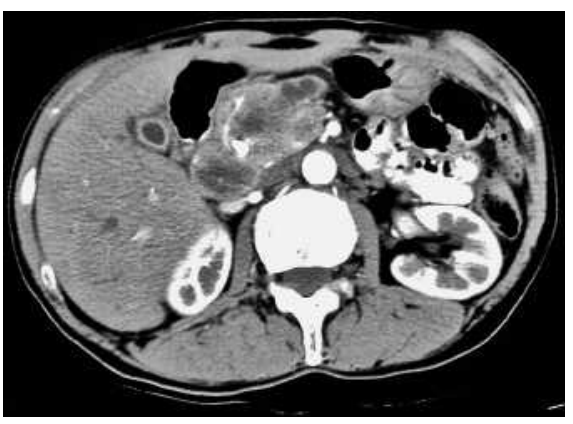

Figure 1 Computed tomographic scan showing an irregularly enhancing mass at the head of the pancreas.

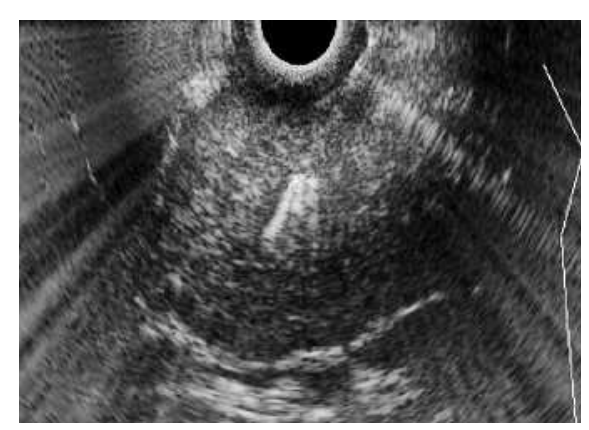

Figure 2 Endoscopic ultrasound-guided fine-needle aspiration of the tumor mass.

lesions, ranging from well- to moderately differentiated tumors (carcinoid or atypical carcinoid) to poorly differentiated carcinoma (small-cell carcinoma), different types sometimes being found within the same tumor [1]. Complete surgical excision is the most important determining factor for predicting long-term survival $[2,3]$. Metastatic spread of thymic cancer to bone, lung, pleura, spleen, brain, and mediastinal lymph nodes has been reported [4]. However, as far as we know, there has only been one case of pancreatic metastasis reported in the literature previously [5]. In the current case, endoscopic ultrasound-guided fine-needle aspiration was shown to be a valuable tool in reaching a diagnosis of this metastatic disease and major surgery was avoided.
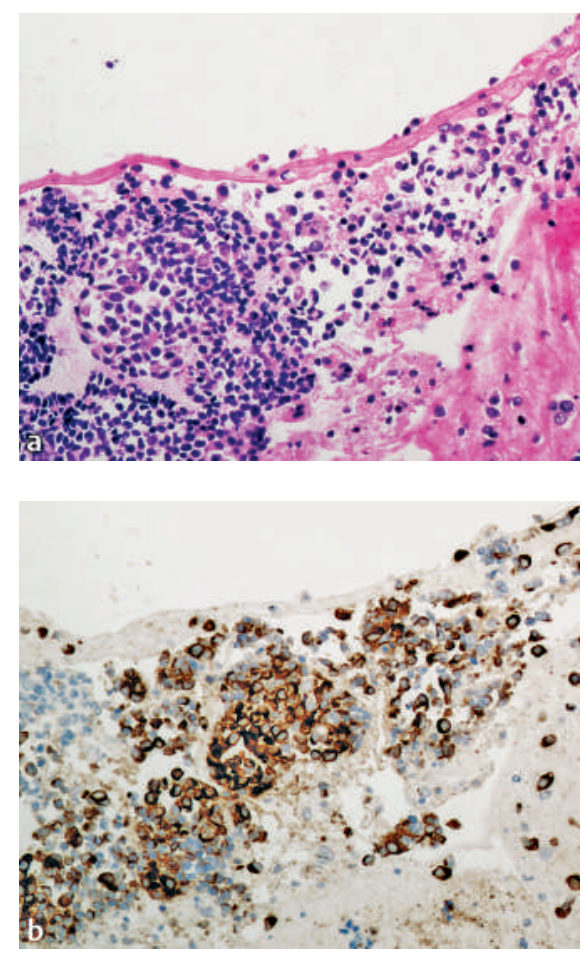

Figure 3 Histological views of the resected tumor. a Cell-block material showing clusters of tumor cells with small hyperchromatic nuclei and a moderate amount of cytoplasm (hematoxylin and eosin stain, magnification $\times 200$ ). b The same clusters of tumor cells after immunohistochemical staining, expressing chromogranin with strong cytoplasmic positivity (chromogranin stain, magnification $\times 200)$.

\section{Y. T. Lee', G. M. Tse ${ }^{2}$, P. B. S. Lai ${ }^{3}$, \\ J. J. Y. Sung1}

${ }^{1}$ Department of Medicine and

Therapeutics, Prince of Wales Hospital, The Chinese University of Hong Kong, Hong Kong

2 Department of Anatomical and Cellular Pathology, Prince of Wales Hospital, The Chinese University of Hong Kong, Hong Kong

${ }^{3}$ Department of Surgery, Prince of Wales Hospital, The Chinese University of Hong Kong, Hong Kong. 


\section{References}

${ }^{1}$ Suster S, Moran CA. Thymoma classification: current status and future trends. Am J Clin Pathol 2006; 125: 542 - 554

2 Suster S, Moran CA. The mediastinum. In: Weidner N, Cote R, Suster S et al (eds). Modern surgical pathology. Philadelphia, Pennsylvania: Saunders 2003: 439-504

${ }^{3}$ Kim DJ, Yang WI, Choi SS et al. Prognostic and clinical relevance of the World Health Organization schema for the classification of thymic epithelial tumors: a clinicopathologic study of 108 patients and literature review. Chest 2005; 127: $755-761$
${ }^{4}$ Fukai I, Masaoka A, Fujii Y et al. Thymic neuroendocrine tumor (thymic carcinoid): a clinicopathologic study in 15 patients. Ann Thorac Surg 1999; 67: 208 -211

${ }^{5}$ Axelson J, Kobari M, Furukawa T et al. Thymic carcinoid in the pancreas: metastatic disease or new primary tumours. Eur J Surg 1999; 165: 270-273

\section{Corresponding Author}

\section{Y. T. Lee, M.D.}

Prince of Wales Hospital

The Chinese University of Hong Kong Shatin, N.T.

Hong Kong SAR

China

Fax: $\quad+852-2637-5396$

E-mail: leeytong@cuhk.edu.hk 